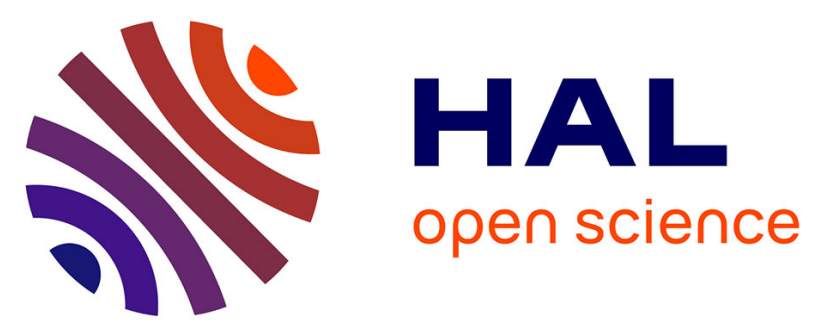

\title{
Adsorption Contraction Mechanics: Understanding Breathing Energetics in Isoreticular Metal-Organic Frameworks
}

Simon Krause, Jack Evans, Volodymyr Bon, Irena Senkovska, Sebastian Ehrling, Ulrich Stoeck, Pascal Yot, Paul Iacomi, Philip Llewellyn, Guillaume Maurin, et al.

\section{To cite this version:}

Simon Krause, Jack Evans, Volodymyr Bon, Irena Senkovska, Sebastian Ehrling, et al.. Adsorption Contraction Mechanics: Understanding Breathing Energetics in Isoreticular Metal-Organic Frameworks. Journal of Physical Chemistry C, 2018, 122 (33), pp.19171-19179. 10.1021/acs.jpcc.8b04549 . hal-01865713

\section{HAL Id: hal-01865713 https://hal.science/hal-01865713}

Submitted on 3 Jul 2019

HAL is a multi-disciplinary open access archive for the deposit and dissemination of scientific research documents, whether they are published or not. The documents may come from teaching and research institutions in France or abroad, or from public or private research centers.
L'archive ouverte pluridisciplinaire HAL, est destinée au dépôt et à la diffusion de documents scientifiques de niveau recherche, publiés ou non, émanant des établissements d'enseignement et de recherche français ou étrangers, des laboratoires publics ou privés. 


\title{
Adsorption Contraction Mechanics: Understanding
} Breathing Energetics in Isoreticular Metal-Organic

\section{Frameworks}

\author{
Simon Krause ${ }^{\ddagger}$, , Jack D. Evans ${ }^{\ddagger, a, b}$,Volodymyr Bon ${ }^{a}$, Irena Senkovska ${ }^{a}$, Sebastian Ehrling ${ }^{a}$, \\ Ulrich Stoeck ${ }^{a}$, Pascal G. Yotc, Paul Iacomi ${ }^{d}$, Philip Llewellyn ${ }^{d}$, Guillaume Maurin ${ }^{c}$, François- $^{\circ}$ \\ Xavier Coudert ${ }^{b}$ and Stefan Kaskel ${ }^{*, a}$
}
${ }^{a}$ Department of Inorganic Chemistry, Technische Universität Dresden, Bergstrasse 66, 01062 Dresden, Germany
${ }^{\mathrm{b}}$ Chimie ParisTech, PSL Research University, CNRS, Institut de Recherche de Chimie, Paris, 75005 Paris, France.

c Institut Charles Gerhardt Montpellier UMR 5253 CNRS UM ENSCM, Université de Montpellier. Place E. Bataillon, 34095 Montpellier cedex 05, France

d Aix-Marseille Univ., CNRS, MADIREL (UMR 7246), 13013 Marseille, France 


\begin{abstract}
A highly porous metal-organic framework DUT-48, isoreticular to DUT-49, is reported with a high surface area of $4560 \mathrm{~m}^{2} \cdot \mathrm{g}^{-1}$ and methane storage capacity up to $0.23 \mathrm{~g} \cdot \mathrm{g}^{-1}\left(164 \mathrm{~cm}^{3} \cdot \mathrm{cm}^{-3}\right)$ at 6.5 $\mathrm{MPa}$ and $298 \mathrm{~K}$. The flexibility of DUT-48 and DUT-49 under external and internal (adsorption-induced) pressure is analyzed and rationalized using a combination of advanced experimental and computational techniques. While both networks undergo a contraction by mechanical pressure, only DUT-49 shows adsorption-induced structural transitions and negative gas adsorption of $n$-butane and nitrogen. This adsorption behavior was analyzed by microcalorimetry measurements and molecular simulations to provide an explanation for the lack of adsorption-induced breathing in DUT-48. It was revealed that for DUT-48 a significantly lower adsorption enthalpy difference and a higher framework stiffness prevent adsorption-induced structural transitions and negative gas adsorption. The mechanical behavior of both DUT-48 and DUT-49 was further analyzed by mercury porosimetry experiments and molecular simulations. Both materials exhibit large volume changes under hydrostatic compression, demonstrating noteworthy potential as shock absorbers with unprecedented high work energies.
\end{abstract}




\section{INTRODUCTION}

Application of pressure to even the hardest materials, such as diamond ${ }^{1}$, will generate changes in structure. For some applications, pressure-induced structural transitions are an important prerequisite, exemplified in zirconia ceramics where crack propagation is stopped by incorporating a metastable phase which undergoes a phase transition to absorb energy. ${ }^{2}$ Moreover, where such transitions are large in magnitude and in a defined area and direction, they can be leveraged for novel shock absorbers. ${ }^{3}$ Amorphous materials often lack these properties due to their disordered structure. Alternatively, many ordered and crystalline materials show defined and large structural changes under the application of an external pressure, however, the prediction and tuning of the transitions remain challenging. Metal-Organic Frameworks (MOFs) are a class of crystalline materials with a wide spectrum of potential applications ${ }^{4}$, in particular for mechanics-related fields. ${ }^{5-6}$ The high porosity in MOFs allows for very large volume changes upon mechanical compression.

Many 'softer' MOFs have shown distinct structural transitions with large changes in structure upon exposure to mechanical pressure ${ }^{7-8}$ but also temperature ${ }^{9}$, irradiation $^{10}$, or to guest molecules. ${ }^{11-12,13}$ Several of these flexible MOFs are excellent candidates for mechanical shock absorbers. ${ }^{14-17,18}$ Under the application of hydrostatic pressure, MIL-53(A1) ${ }^{3}$ (MIL = Material of Institute Lavoisier) and MIL-47(V) ${ }^{17}$ can switch from a large pore to a contracted pore state. Due to this large change in unit cell volume $(\sim 35 \%)$ combined with the high pressure required to induce the structural transition $(\sim 120 \mathrm{MPa}), \mathrm{MIL}-47(\mathrm{~V})$ is regarded as one of the best shock absorber materials. More recently, the fumarate version of MIL-53 in its Ga and Al forms has shown even higher mechanical energy absorption performances. ${ }^{3,16}$ Moreover, adsorption-induced stress, 
which acts similarly to internal pressure ${ }^{19-20}$, can also cause contraction of MIL-53. ${ }^{21-22}$ Upon exposure to guest molecules such as water, methane, carbon dioxide or xenon, the guest-free framework contracts at low pressures and expands again at higher pressures to its original state, filled with guest molecules. This transition which is also referred to as breathing can be also manipulated by functionalization of the terephthalate linker. ${ }^{23}$

An entirely new type of breathing transition in a mesoporous MOF has been recently observed in DUT-49 (DUT = Dresden University of Technology), which contracts from an open pore (op) structure to a contracted pore $(c p)$ form under adsorption-induced stress of methane, $n$-butane ${ }^{24}$ or xenon. ${ }^{25}$ A novel aspect of this mesoporous breathing MOF is the passage through a metastable state during adsorption causing a counterintuitive phenomenon called negative gas adsorption (NGA). With increasing gas pressure, DUT-49 releases previously adsorbed gas from its pore upon structural contraction in the $c p$ state causing a negative step in the isotherm $\left(\partial \mathrm{N}_{\mathrm{ads}} / \partial \mathrm{P}<0\right)$. The NGA phenomenon implies the material acts as a pressure amplifier, which is an interesting conceptually novel function of adsorbents. The structural transitions responsible for the NGA behavior have been extensively investigated by in situ diffraction and spectroscopic methods ${ }^{24}$ as well as theoretical calculations. ${ }^{26}$ However, the question of the molecular origins and construction principles of such pressure amplifying materials is still an open one. In particular the development of rational concepts to further extend this class of materials requires a better understanding of their intrinsic mechanics for tuning NGA and thus mechanical energy storage in materials.

In this contribution we report DUT-48 as a highly porous MOF isoreticular to DUT-49 with reduced ligand length (9,9'-(1,4-phenylene)bis(9H-carbazole-3,6-dicarboxylate,cpcdc) (Scheme 1). A series of experimental techniques including adsorption, microcalorimetry and mercury 
intrusion were coupled to explore the guest and mechanically-induced flexible behavior of DUT48. An important finding is that both DUT-48 and DUT-49 materials can be termed as "flexible" MOFs since both materials undergo structural transformations under external pressure, showing potential as shock absorbing materials. However, the enhanced stiffness of DUT-48 caused by the shorter linker prevents adsorption-induced breathing. This difference in behavior was further interpreted and rationalized by a combination of force field and quantum-based molecular simulations.

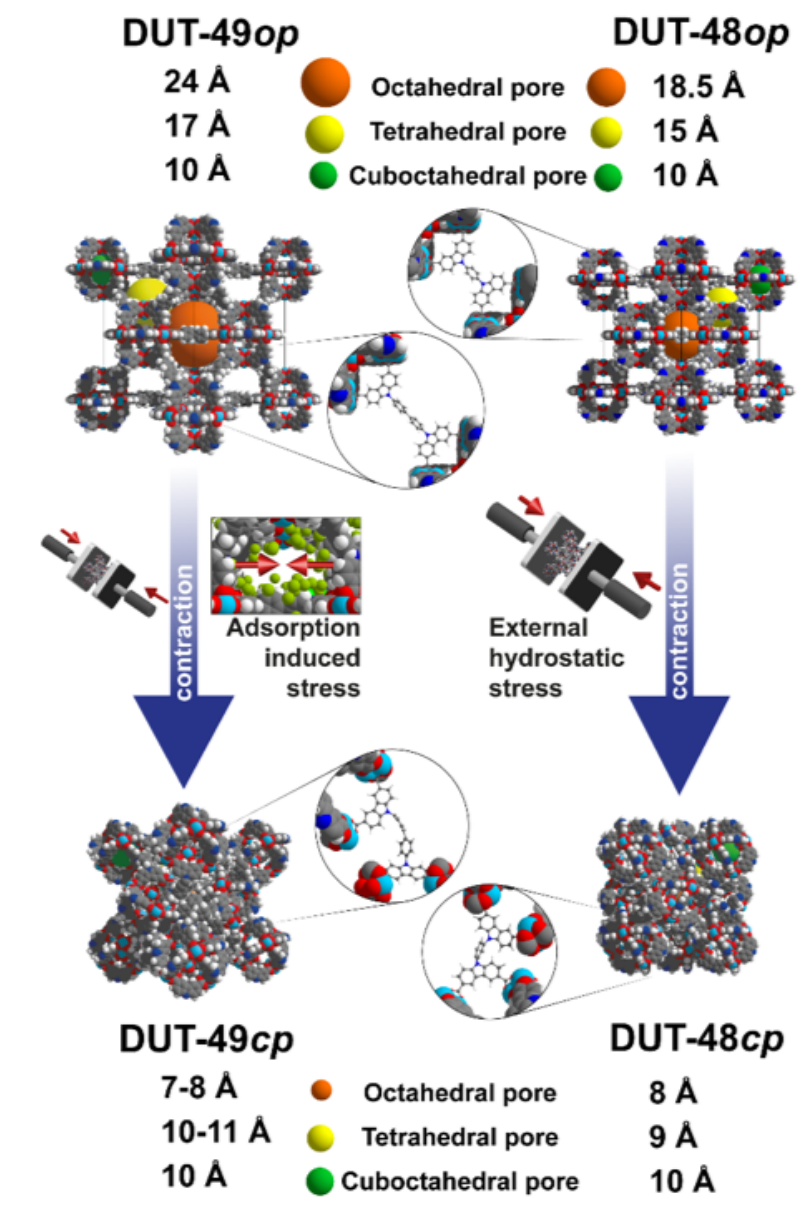

Scheme 1. Comparison of DUT-49op and DUT-48op framework and pore size including magnified illustration of linker molecules. Structural contraction induced by either external hydrostatic compression or internal adsorption induced stress to the contracted phases DUT-49cp and DUT-48cp. 


\section{EXPERIMENTAL AND THEORETICAL METHODS}

\section{Synthesis of organic ligands and MOF materials}

The ligand (9,9'-([1,1'-biphenyl]-4,4'-diyl)bis(9H-carbazole-3,6-dicarboxylic acid)) (H4bbcdc) was synthesized according to a previously reported procedure. ${ }^{24}$ The Ligand $\mathrm{H}_{4} \mathrm{cpcdc}$ was synthesized according to the procedure used for a similar carbazole-containing ligand. ${ }^{27}$ Both MOF materials were synthesized according to a previously mentioned procedure. ${ }^{24,28}$ Detailed synthesis procedures, activation of the MOF materials and characterization are provided in the ESI.

\section{Gas adsorption experiments}

Low pressure adsorption experiments were performed on a BELSORP-max, high pressure adsorption on a BELSORP-HP instrument. Gases of purity $99.9 \%$ or higher were used. Detailed information about the adsorption measurements are provided in the ESI.

\section{Microcalorimetry experiments}

Isotherms and corresponding enthalpies were measured using a Tian-Calvet type microcalorimeter coupled with a home-made manometric gas dosing system. ${ }^{29}$ This apparatus allows for the simultaneous measurement of the adsorption isotherm and corresponding differential enthalpies. Further details are provided in the ESI.

\section{Intrusion experiments}


Mercury intrusion experiments were carried out in the range of pressure atm. - $415 \mathrm{MPa}$ available with the Hg-porosimeter Micromeritics Autopore IV 9500. Details of sample preparation are provided in the ESI.

\section{Crystallography}

A cubic single crystal of DUT- 48 was placed into a glass capillary $(\mathrm{d}=0.3 \mathrm{~mm})$ with small amount of the mother liqueur. Data was collected at BL14.2 beamline of the MX-facility at BESSY-II synchrotron, operated by Helmholtz-Zentrum Berlin für Materialien und Energie. Single crystal structure of DUT-48: $\mathrm{C}_{34} \mathrm{H}_{20} \mathrm{Cu}_{2} \mathrm{~N}_{2} \mathrm{O}_{10}, 743.6 \mathrm{~g} \mathrm{~mol}^{-1}$, cubic, $\mathrm{Fm} \overline{3} \mathrm{~m}, \mathrm{a}=40.490$ (5) $\AA, \mathrm{V}=66381(23) \AA^{3}, \mathrm{Z}=24, \rho c a l=0.444 \mathrm{~g} \cdot \mathrm{cm}^{-3}, 3870$ independent reflections observed, $\mathrm{R} 1=$ $0.0748[\mathrm{I}>2 \sigma(\mathrm{I})], \mathrm{wR} 2=0.2286$ (all data) and GoF 1.121. This data can be obtained free of charge from the Cambridge Crystallographic Data Centre under entry CCDC-1827897 via www.ccdc.cam.ac.uk/data_request/cif. Additional experimental details can be obtained from ESI.

\section{Simulation Details}

The behavior and mechanical properties of the representative ligands were investigated for the corresponding acid via Density Functional Theory(DFT) optimizations using the CRYSTAL14 software ${ }^{30}$ with localized TZVP basis sets ${ }^{31}$ and the hybrid exchange-correlation functional PBE0 $0^{32}$ Long-range dispersion corrections were included via the Grimme 'D2" approach ${ }^{33}$ Stress-strain

curves were subsequently produced using the methodology described previously ${ }^{26}$ Grand Canonical Monte Carlo(GCMC) simulations were employed to simulate the adsorption and 
enthalpy of $n$-butane using the RASPA2.0 code. $^{34}$ Frameworks were treated by the UFF forcefield ${ }^{35}$ and $n$-butane the united-atom TraPPe force field ${ }^{36}$

Molecular dynamics (MD) simulations used a modified MOF-FF force field ${ }^{37}$ recently adapted to lammps ${ }^{38}$ by Boyd et al..$^{39}$ to describe the bonds, angles, dihedrals and improper dihedrals present in the DUT-48 and DUT-49 frameworks. Further details can be found in the supporting information. Representative input files for molecular simulations are available online in our data repository at https://github.com/fxcoudert/citable-data. 


\section{RESULT AND DISCUSSION}

The ligand $\mathrm{H}_{4}$ cpcdc (Scheme 1) used for the synthesis of DUT-48 was obtained in a large-scale 5-step synthesis with high yield based on simple starting materials and $9 H$-carbazole (synthetic protocol in ESI). The ligand was used in a solvothermal reaction with $\mathrm{Cu}\left(\mathrm{NO}_{3}\right)_{2} \cdot 3 \mathrm{H}_{2} \mathrm{O}$ in DMF or NMP to yield DUT-48 as blue powder. Zhou and co-workers have reported the synthesis of PCN81, isomorphous to DUT-48 but crystallizing in the cubic space group $P a \overline{3}$, and PCN-82, bearing two additional methoxy groups on the linker and crystalizing in $F m \overline{3} m .{ }^{28}$ However, attempts to reproduce the reported structure of $\mathrm{PCN}-81$ by replicating and also modifying the reaction conditions (see ESI) always yielded phase pure DUT-48, crystalizing in cubic space group $F m \overline{3} m$. From three different synthesis protocols the reaction of $\mathrm{H}_{4}$ cpcdc with $\mathrm{Cu}\left(\mathrm{NO}_{3}\right)_{2} \cdot 3 \mathrm{H}_{2} \mathrm{O}$ in $\mathrm{DMF}$ with acetic acid as modulator (for details see ESI) yielded the best result and this was thus used for the further experiments. The structure of DUT-48 was determined by synchrotron based single crystal diffraction experiments (see Tab. S2). DUT-48 is isoreticular and isosymmetrical to DUT49, while the linker is slightly buckled in PCN-81 (Fig. S9). PCN-82 in contrast to PCN-81 retains its structure upon removal of solvent by freeze-drying and shows permanent porosity for gases. ${ }^{28}$

To analyze the adsorption behavior of DUT-48 a well-established activation protocol for removal of solvent from the pores, involving supercritical $\mathrm{CO}_{2}$ drying, was applied. ${ }^{27}, 40$ The resulting deep blue powder was activated additionally at $120^{\circ} \mathrm{C}$ in dynamic vacuum $\left(\mathrm{p}<10^{-3}\right.$ mbar). Powder X-ray diffraction (PXRD) analysis showed structural preservation of DUT-48 upon activation. It is thermally stable up to $350^{\circ} \mathrm{C}$ similar to DUT-49, as determined by thermogravimetric analysis (Fig S10). 
The pore volume and apparent BET area of DUT-48 were determined on the basis of a $\mathrm{N}_{2}$ adsorption isotherm at $77 \mathrm{~K}$. A high BET area of $4560 \mathrm{~m}^{2} \cdot \mathrm{g}^{-1}$ and specific pore volume of 1.98 $\mathrm{cm}^{3} \cdot \mathrm{g}^{-1}$ could be determined improving values for isoreticular PCN-82 $\left(4480 \mathrm{~m}^{2} \cdot \mathrm{g}^{-1}, 1.7 \mathrm{~cm}^{3} \cdot \mathrm{g}^{-1}\right)^{28}$ but lower compared to DUT-49 $\left(5476 \mathrm{~m}^{2} \cdot \mathrm{g}^{-1}, 2.68 \mathrm{~cm}^{3} \cdot \mathrm{g}^{-1}\right)^{41}$ due to the reduced pore size.

The high BET area and specific pore volume motivated us to investigate the storage capacity of $\mathrm{CH}_{4}, \mathrm{H}_{2}$ and $\mathrm{CO}_{2}$ at high pressures (ESI). As expected, the maximum gravimetric excess uptake $\left(\mathrm{CH}_{4} 0.23 \mathrm{~g} \cdot \mathrm{g}^{-1}, \mathrm{CO}_{2} 1.23 \mathrm{~g} \cdot \mathrm{g}^{-1} ; \mathrm{H}_{2} 80 \mathrm{mg} \cdot \mathrm{g}^{-1}\right)$, as well as the pressure, at which the maximum capacity is reached $\left(\mathrm{CH}_{4} 100\right.$ bar, $\mathrm{CO}_{2} 45$ bar; $\mathrm{H}_{2} 50$ bar) are lower for DUT-48 in comparison to DUT-49 due to the reduced porosity. The total $\mathrm{CH}_{4}$ adsorption capacity at $6.5 \mathrm{MPa}$ and $25^{\circ} \mathrm{C}$ is $164 \mathrm{~cm}^{3} \cdot \mathrm{cm}^{-3}\left(0.27 \mathrm{~g} \cdot \mathrm{g}^{-1}\right)$.

Neither the high-pressure adsorption experiments nor the $\mathrm{N}_{2}$ adsorption isotherm at $77 \mathrm{~K}$ showed hysteresis or unusual steps as indications for structural transitions similar to DUT-49 (Figure 1). From our previous studies of DUT-49 it is known that adsorption-induced transitions occur at temperatures close to the respective boiling point of the adsorbate. Nitrogen at $77 \mathrm{~K}$ was found to be a very sensitive adsorbate only initiating structural transitions for DUT-49 crystals larger than $1-2 \mu \mathrm{m} .{ }^{42}$ Consequently, adsorption isotherms close to ambient temperature were recorded using $n$-butane at the respective boiling point of $273 \mathrm{~K}$ and also at $295 \mathrm{~K}$ and $303 \mathrm{~K}$ (Figure 1 and Figure 2). These conditions were previously found to induce a contraction from DUT-49op to DUT-49cp independent of particle size, which has been well studied by in situ PXRD in parallel to adsorption and derived from multiple steps, hysteresis and spikes in the adsorption isotherms. ${ }^{24}$ 

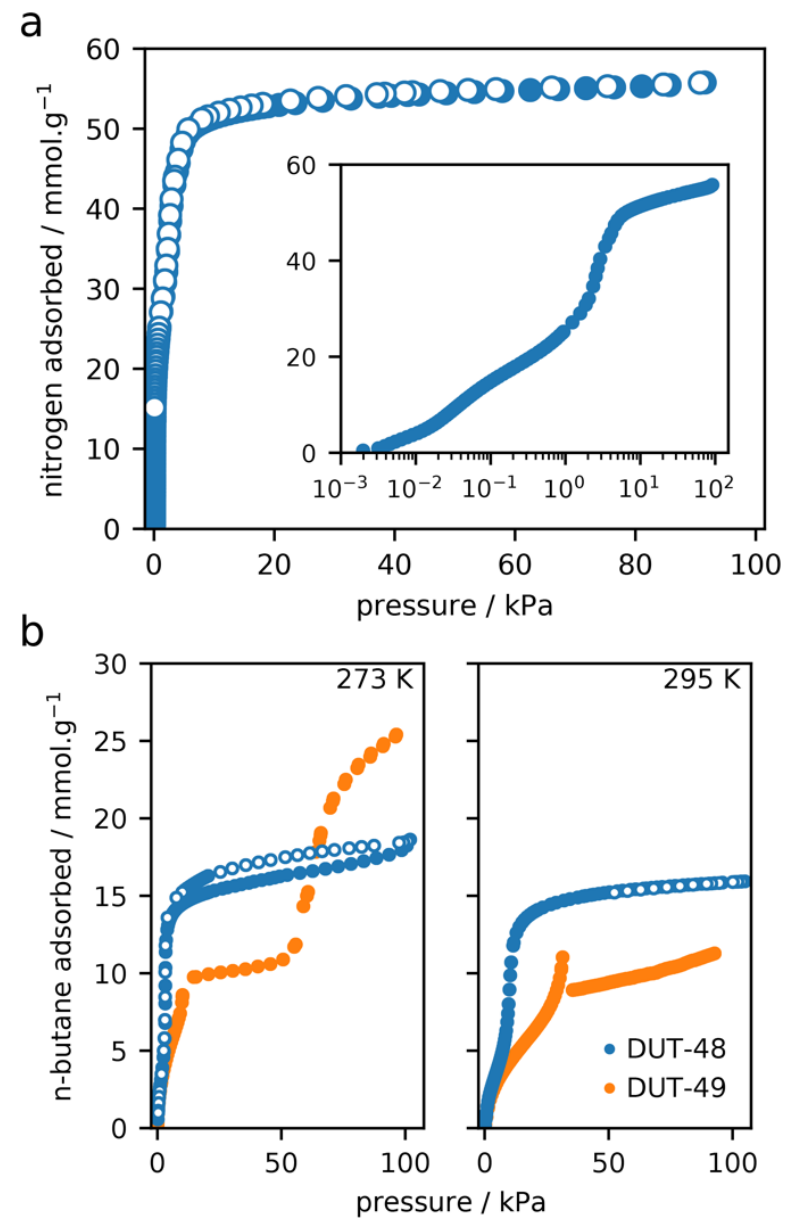

Figure 1. a) Nitrogen adsorption isotherm at $77 \mathrm{~K}$ on DUT-48, inset semilogarithmic. b) $n$-butane adsorption isotherm at $273 \mathrm{~K}$ and $295 \mathrm{~K}$. Isotherms of DUT-48 and DUT-49 are displayed in blue and orange circles, respectively. Adsorption and desorption are displayed as filled and open symbols, respectively. 
In the case of DUT-48 neither adsorption of nitrogen or $n$-butane induced a structural transition, illustrated by the lack of steps or hysteresis in the isotherms (Figure 1). PXRD measurements after the adsorption experiments showed no sign of structural transitions or decomposition of the pristine material, in contrast to DUT-49 (Fig. S9).

\section{Analysis of Adsorption and Structure Thermodynamics}

Upon contraction in DUT-49 the adsorption enthalpy for $n$-butane rises from $29.4 \mathrm{~kJ}^{\mathrm{mol}}{ }^{-1}$ for the $o p$ structure to over $50 \mathrm{~kJ} \cdot \mathrm{mol}^{-1}$ for the $c p$ structure, due to the reduction of porosity and increased gas-surface interactions. ${ }^{24}$ This increase in adsorption enthalpy which was calculated in our original study for DUT-49 as $\Delta \Delta H=153 \mathrm{~kJ} \mathrm{~mol}^{-1}$ per ligand compensates for the energy required for the endothermal structural transition that occurs upon NGA. ${ }^{24}$

To investigate the effect of a smaller pore structure we monitored the pressure dependence of the adsorption enthalpies for $n$-butane at $303 \mathrm{~K}$ for both DUT-48 and DUT-49 (Figure 2). ${ }^{29}$ This provides access to experimental isosteric $\Delta H_{\text {ads }}$ of the $c p$ phase in DUT-49 as well as the full profile for DUT-48. The experimentally obtained enthalpies match the values calculated with the Van't Hoff equation well (see ESI) indicating the validity of the data. A large increase in enthalpy is observed for DUT-49 after NGA due to the stronger interactions of $n$-butane with the microporous $c p$ phase of DUT-49. However, we are unable to measure the enthalpies of adsorption for both, the $o p$ and $c p$ phases because the $o p$ and especially the $c p$ phase is only experimentally present in a constrained pressure region. Thus, enthalpies of adsorption for $o p$ - and $c p$ phases of DUT-49 and DUT-48 were calculated for adsorption of $n$-butane using energy/particle fluctuations with GCMC methods (Figure 2). A representative model for a $c p$-phase of DUT-48 was generated from forcefield based MD simulations (additional details for the model of DUT-48cp are described later). 
a

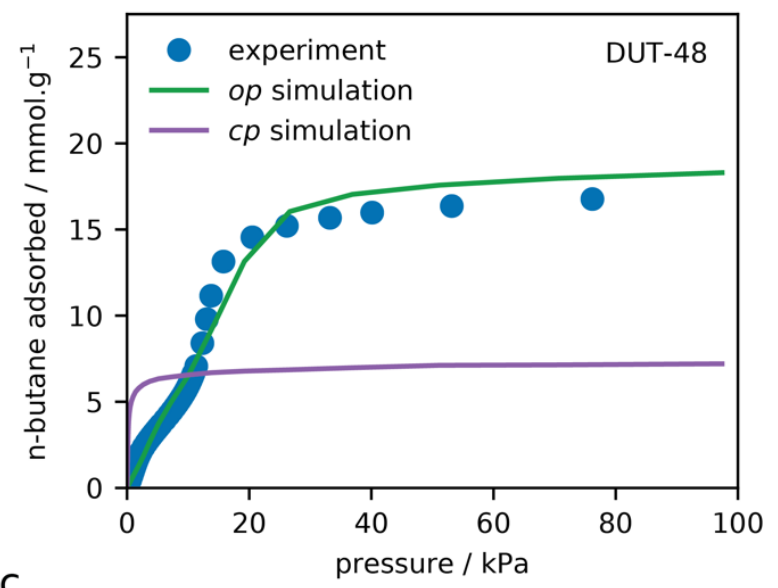

$\mathrm{C}$

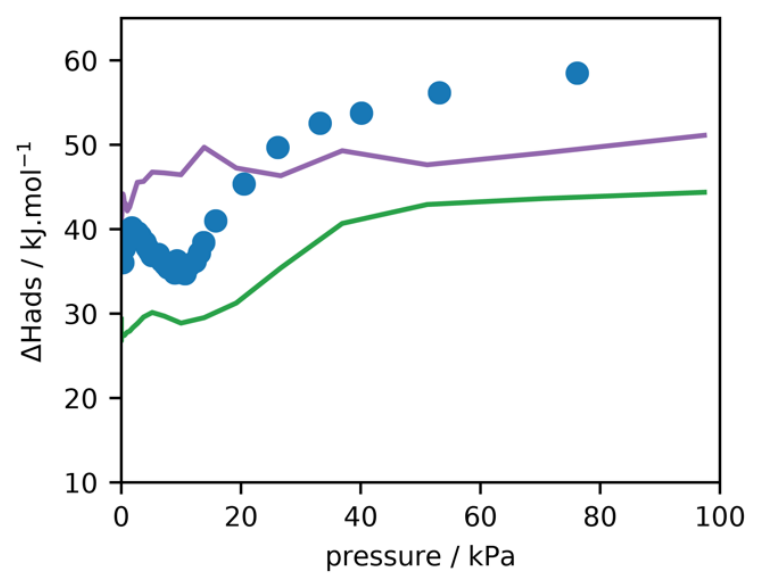

b

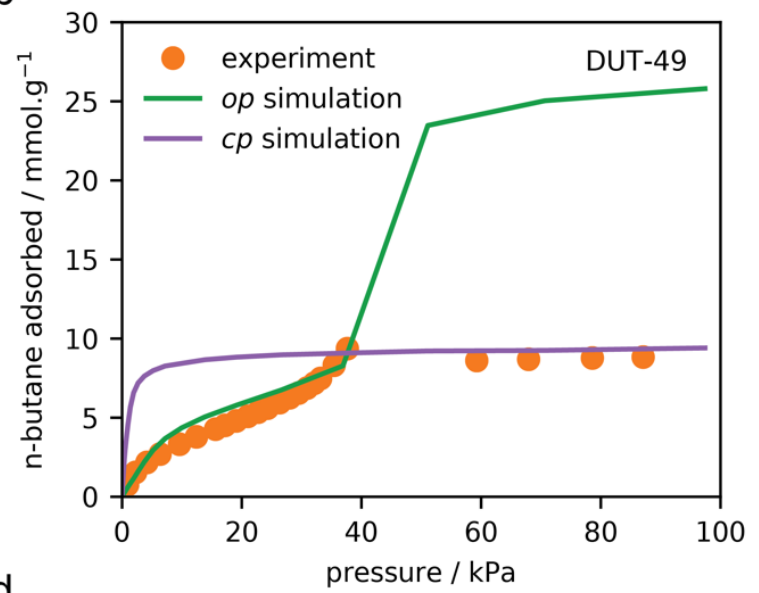

d

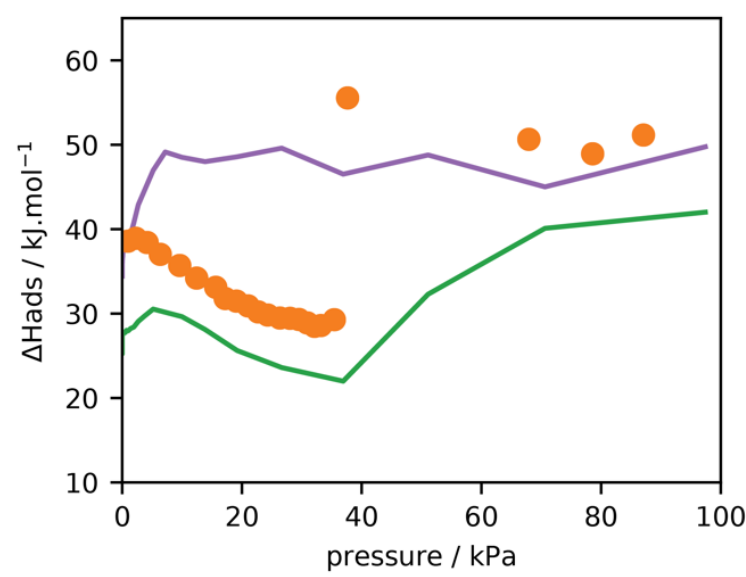

Figure 2. Experimental $n$-butane adsorption isotherm of a) DUT-48 and b) DUT-49 at 303 K (blue circles) including GCMC simulations for the predicted $o p$ - and $c p$-phases (green and purple, respectively). Adsorption enthalpies for $n$-butane in c) DUT-48 and d) DUT-49 determined by calorimetry (blue circles), calculated by GCMC simulations for open pore (green line) and contracted pore (purple line) structures.

Simulated adsorption enthalpies track well with the experimental values allowing for an accurate analysis of the adsorption enthalpies for all structures involved. There is a systematic underestimation of $\Delta H_{\text {ads }}$ by simulation by ca. $20 \%$ as sampling the energetics of flexible $n$-butane is challenging by Monte Carlo methods - we note here that we used a readily available force field with no adjustment. The experimental $\Delta H_{\text {ads }}$ of DUT-49 shows a clear discontinuity after NGA 
with a significant increase in adsorption enthalpy. This change corresponds well to the disparate GCMC adsorption enthalpies calculated for the $o p$ and $c p$ phases $\left(43.7\right.$ and $21.6 \mathrm{~kJ}^{\mathrm{mol}}{ }^{-1}$, respectively). The $c p$-phase of DUT-49 has a significantly higher adsorption enthalpy owing to the drastic reduction in pore size that leads to a significant increase of the host/guest interactions.

Contrastingly, the adsorption enthalpy of DUT-48 follows the behavior of the $o p$ phase corresponding to the behavior expected for a nanoporous material matching the profile predicted by GCMC simulations. This behavior can be characterized by an initial increase associated with cooperative adsorption at favorable adsorption sites in the smallest cubaoctahedral pores followed by a decrease once these pores are filled and subsequently upon similar cooperate adsorption in the larger pores there is an increase. Notably, simulation reveals this final increase occurs at lower pressure in DUT-48 than DUT-49 due to a smaller large pore size. The values obtained from, in situ experiment and GCMC simulations are summarized in Table 1.

Table 1. Summary of $\Delta H_{\text {ads }}$ and $\Delta \Delta H_{\text {ads }}$ values determined by, microcalorimetry, calculation using equation (1), and GCMC simulations for $o p$ and $c p$ structures of DUT-48 and DUT-49 at 10 and $39 \mathrm{kPa}$, during $n$-butane adsorption at $303 \mathrm{~K}$. Van't Hoff values were determined at loadings of 6.5 and $8.8 \mathrm{mmol.g} \mathrm{g}^{-1}$, respectively. Uptakes for calculation of $\Delta \Delta H_{\text {ads }}$ were 110 and 166 molecules $n$-butane per unit cell. $\Delta \Delta H_{\text {ads }}$ values are given per ligand with 24 ligands per unit cell.

\begin{tabular}{llll}
\hline & $\begin{array}{l}\text { Microcalorimetry } \\
\left(\mathrm{kJ} \cdot \mathrm{mol}^{-1}\right)\end{array}$ & Van’t Hoff & GCMC \\
\hline DUT-48op & 34.6 & 34.8 & 27.3 \\
DUT-48cp & n. a. & n. a. & 45.0 \\
DUT-49op & 29.4 & 26.5 & 21.6 \\
DUT-49 $c p$ & $51( \pm 4)$ & n.a. & 43.7
\end{tabular}



$\Delta \Delta H_{\text {ads }}$ DUT-48
n. a.
n. a.
81

$\Delta \Delta H_{\text {ads }}$ DUT-49

149 $( \pm 26)$

n. a.

153

The simulated adsorption enthalpy for the $o p$ - and $c p$-phases can be used to determine the energy gain, $\Delta \Delta H_{\text {ads }}$ (Equation 1), for a transition from $o p$ - to $c p$-phase.

$\Delta \Delta H_{a d s}=n_{a d s}(c p) \cdot\left|\Delta H_{a d s}(o p)-\Delta H_{a d s}(c p)\right|$

In agreement with the previous study ${ }^{24}$, we find $\Delta \Delta H_{\text {ads }}$ for $n$-butane and DUT-49 at $\sim 39 \mathrm{kPa}$ and $303 \mathrm{~K}$ to be at around $150 \mathrm{~kJ} \cdot \mathrm{mol}^{-1}$ per ligand. In contrast for DUT-48, at $\sim 10 \mathrm{kPa}$, the potential energy gain is around $80 \mathrm{~kJ} . \mathrm{mol}^{-1}$, significantly lower than DUT-49.

In addition to understanding the thermodynamics of adsorption, we have applied molecular simulations to examine the thermodynamics of the DUT-48 framework itself. Total energies were calculated using several constant-volume $(\mathrm{N}, \mathrm{V}, \mathrm{T}) \mathrm{MD}$ simulations and free energies were calculated using thermodynamic integration. ${ }^{43}$ The resulting energy profiles show two minima for both DUT-49 and DUT-48 (Figure 3), as expected for bistable breathing MOFs. ${ }^{19}$ The minima at large volumes correspond to the observed crystal structure of the materials (op-phase). The second minimum, at small cell volumes, owing to the conformational strain on the ligand is greater in energy than the $o p$-phase. Importantly, these simulations highlight that a $c p$-phase for DUT-48 exists with a cell parameter of $32.8 \AA$, compared to $40.5 \AA$ for the experimental $o p$-phase. The $c p$ phase for DUT-48 is approximately $5 \mathrm{~kJ} \mathrm{~mol}^{-1}$ per ligand higher in energy than for DUT-49. Additionally, the energy barrier for the $o p$ - $c p$ transition is also greater. 


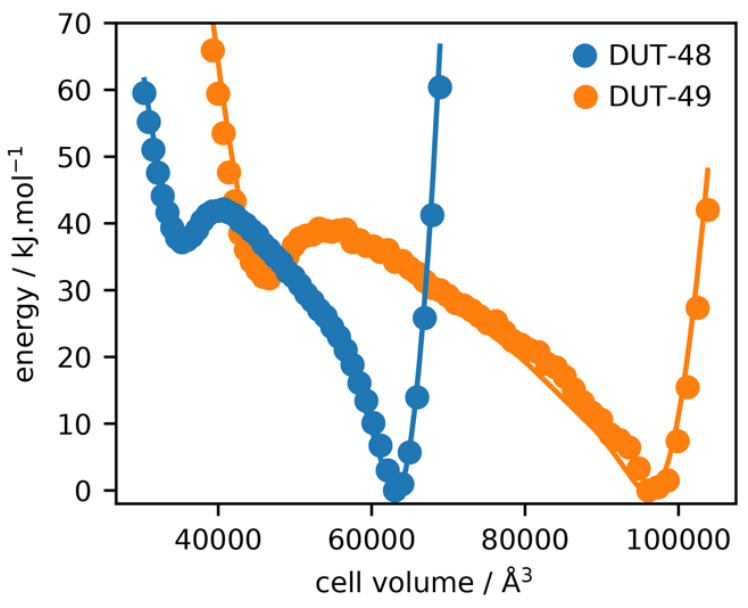

Figure 3. Profiles Free and internal energy (solid line and coloured circles) obtained from (N, V, T) simulations as a function of unit-cell volume at $300 \mathrm{~K}$. Energy is displayed per ligand. 
The comprehensive thermodynamic analysis described here exposes two key differences between DUT-48 and DUT-49 that prevent DUT-48 from undergoing an adsorption-induced transition to a $c p$ phase:

Firstly, the differences in adsorption enthalpies ( $\left.\Delta \Delta H_{\text {ads }}\right)$ are significantly smaller than observed for DUT-49. Secondly, the proposed $c p$-phase requires a greater unfavorable strain of the building units resulting in a significantly higher energy state. Although the combination of these two factors results in the rigid adsorption profiles observed for DUT-48. We expect that these factors can be tuned using alternative ligands to produce a stimuli-responsive framework and thus providing the basis for further modification of DUT-49.

\section{Analysis of Mechanical Stability}

The adsorption-induced stress on the framework is limited by the extent of the host-guest interactions, as described above. As a result, the presented adsorption experiments for DUT-48 exhibit no structural contraction as the stress is not large enough to compensate for the energy required for a transition to the $c p$-phase, which presence is indicated by the MD simulations. Thus, we further investigated the mechanical behavior of DUT-48 and DUT-49 in the absence of adsorbates to analyze if it is possible to contract DUT-48 by external stress, i.e. applying mechanical pressure.

Crystal structures of DUT-49, op and $c p$ feature a copper paddlewheel environment that is mostly undeformed in the $c p$ structure. ${ }^{24,}{ }^{26}$ However, the ligand is significantly bent with deformation localized to the biphenyl unit, showing considerable similarity to the buckling behavior of macroscopic columns under load. ${ }^{44}$ DFT optimizations of the single isolated building unit with a series of fixed decreasing $\mathrm{N}$-atom-to-N atom $(\mathrm{N}-\mathrm{N})$ distances has provided excellent 
insight into the mechanical force required to produce the $o p-c p$ transition in DUT-49. We have employed these simulations for the ligands used to form DUT-48 and DUT-49 and the resulting energy-strain curve was used to obtain the stress-strain profiles depicted in Figure 4. The bending of the ligands can be characterized by a 'deflection' parameter, which is the N-centroid-N angle. The ligands exhibit two distinct regimes with the application of strain.
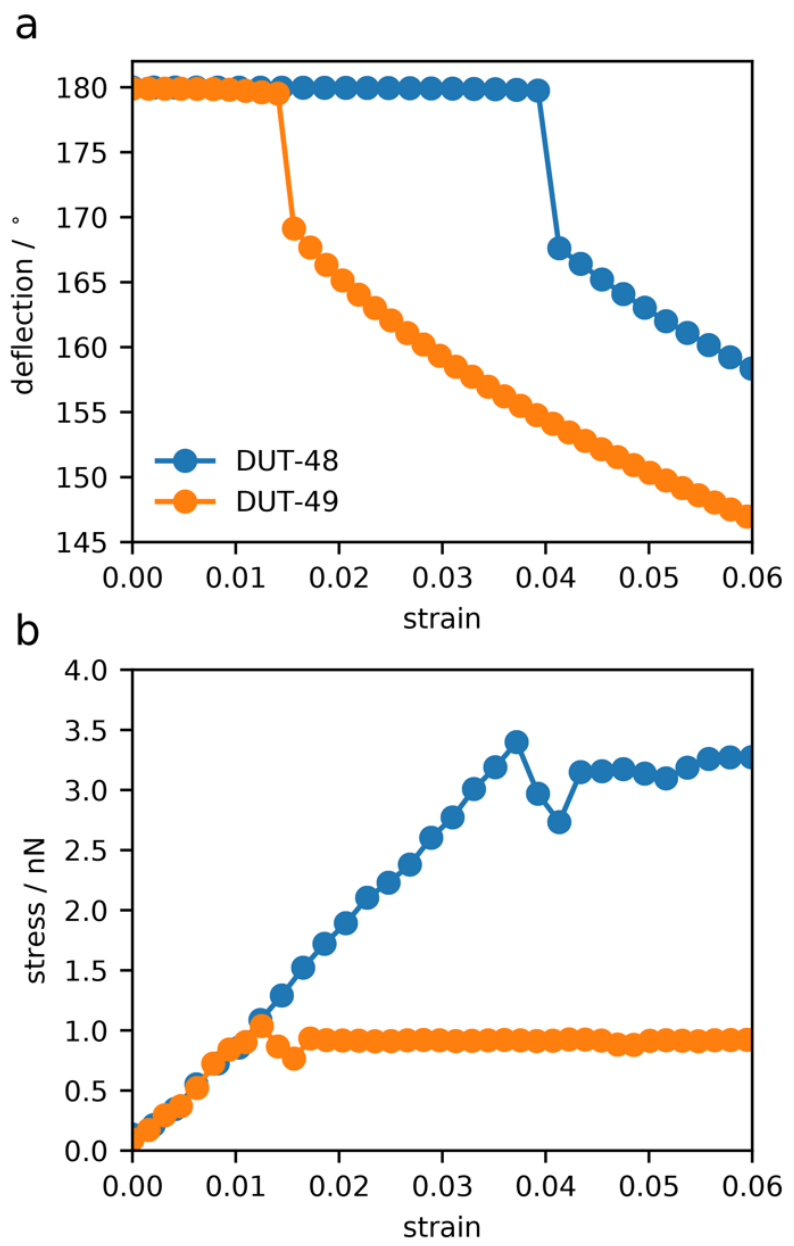

Figure 4. a) Deflection angle for the ligands used to produce DUT-48 and DUT-49, defined as the N-centroid-N angle, for increasing strain. b) Stress-strain curve of the ligands with increasing axial compression. 
Firstly, the elastic regime corresponds to small deformations resulting in no deflection to the ligand. However, larger deformations produce a buckling phenomenon resulting in a sudden large deflection of the ligand and distortions of other key bonds lengths and angles illustrated in the supporting information. We find here that the smaller ligand of DUT-48 requires almost three times the pressure to produce the buckling event than DUT-49. We note that this result is expected from Euler's column formula where the stress required to produce buckling is inversely proportional to the length of the column. ${ }^{45}$ Nevertheless, these results pertain to only a single molecule. To investigate the response of the entire periodic lattice we consult the constant-volume $(\mathrm{N}, \mathrm{V}, \mathrm{T}) \mathrm{MD}$ simulations discussed previously (Figure 5).

Notably, the pressure-profiles resulting from these simulations confirm the conclusions gained from the study of the adsorption case. DUT-48 requires a transition pressure of $\sim 150 \mathrm{MPa}$ significantly greater than that of DUT-49, $60 \mathrm{MPa}$. This transition pressure, while greater than DUT-49, is similar to other flexible frameworks and is achievable experimentally by applying hydrostatic pressure to provide an alternative pathway to adsorbate-induced $o p$ - $c p$ transition.

The pressure-induced structural behavior of DUT-48 and DUT-49 was further explored by mercury intrusion which has been established to ensure application of pressure under hydrostatic conditions. ${ }^{8,17}$ In addition, $\mathrm{Hg}$ is a non-wetting fluid that is expected to not enter mesopores in the range of pressure [0-400 MPa] following the Washburn equation to enable the mechanical stability of the evacuated MOF framework. While this structural deformation is a very different mechanism to that attributed to adsorption we hypothesize the response of the materials are similar. Figure $5 \mathrm{~b}$ reports the evolution of the cumulative intruded mercury as a function of the applied pressure for two cycles of compression-decompression. 
a

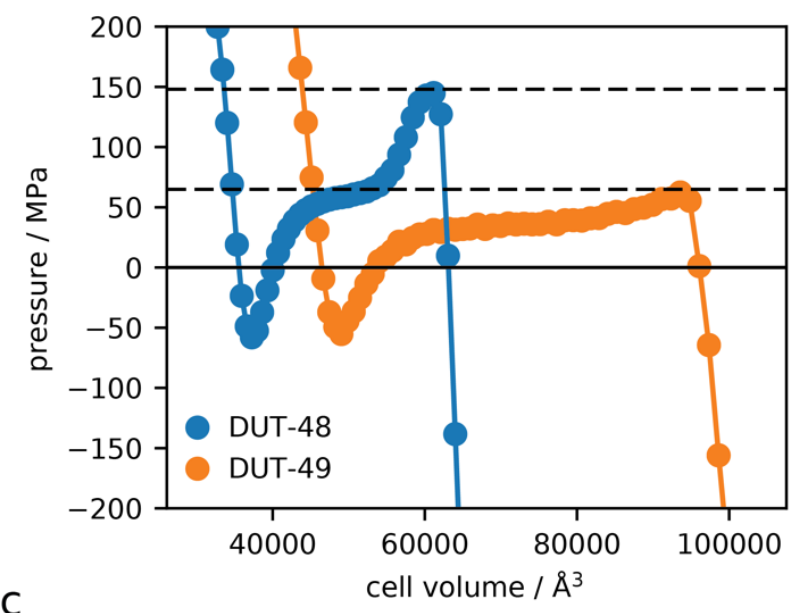

$\mathrm{C}$

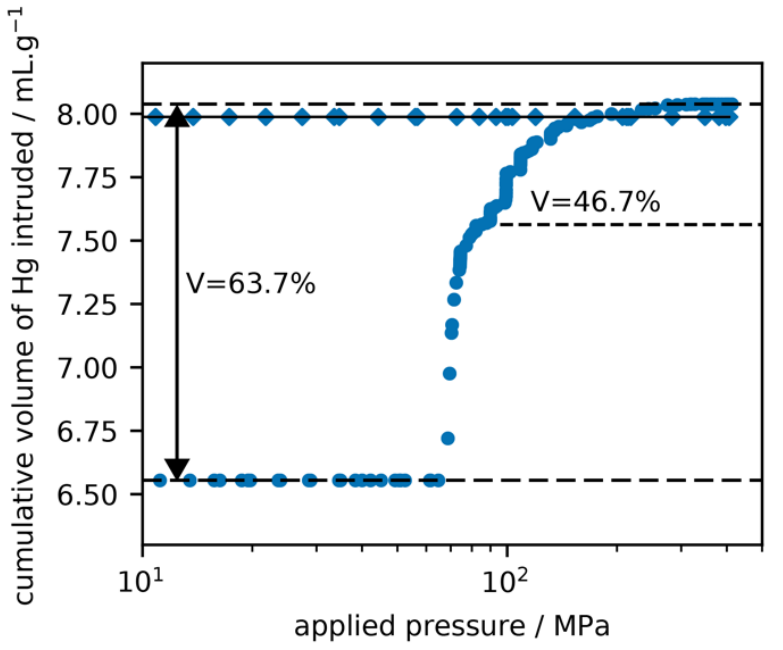

$b$

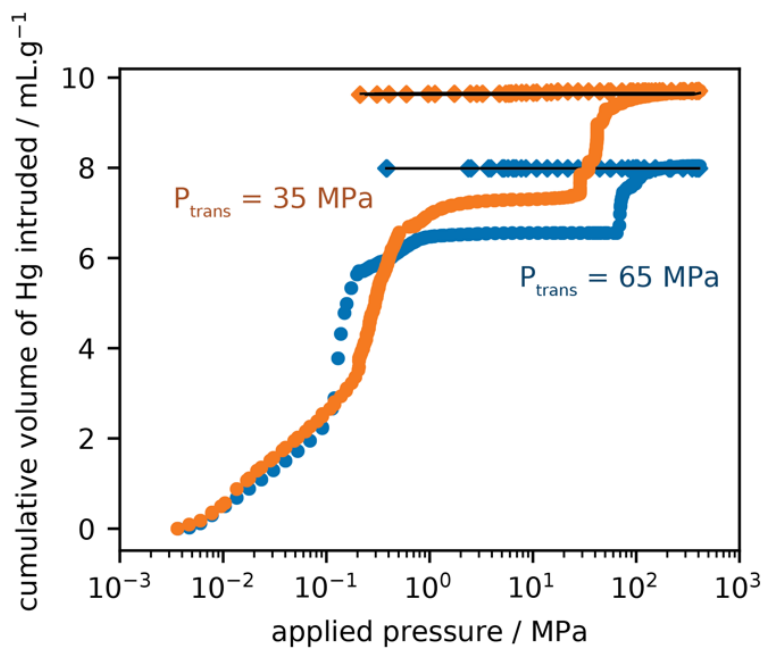

d

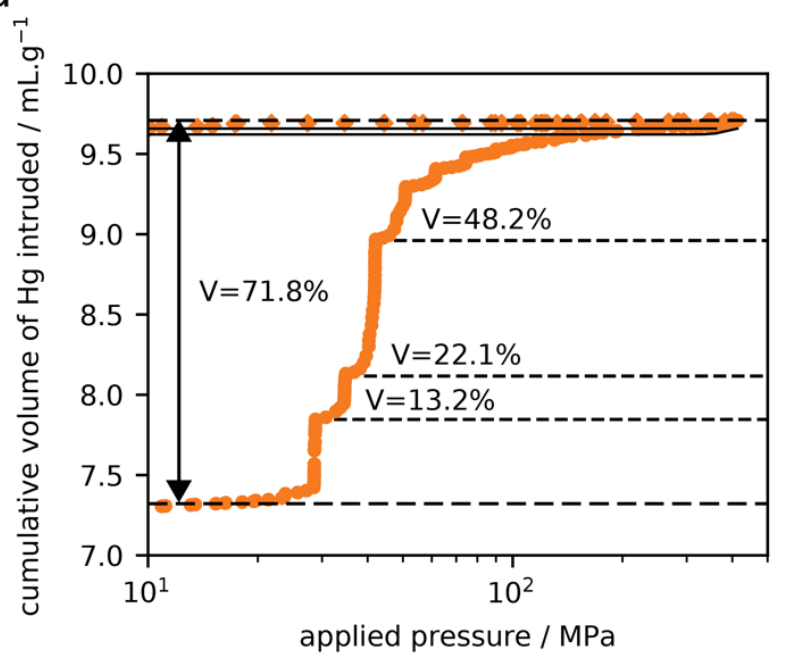

Figure 5. a) Profiles of internal pressure for DUT-48 and DUT-49 obtained from (N, V, T) molecular dynamics simulations as a function of unit cell volume at $300 \mathrm{~K}$. The transition pressure for an $o p \rightarrow c p$ transition is marked by a dotted line. b) Cumulative volume of intruded mercury in two intrusion/extrusion cycles as a function of the applied pressure for the activated DUT48 and DUT49 (blue and orange, respectively). c-d) Gradual volume variation of mercury intruded during the contraction show the difference in behavior for DUT48 two steps (c) and DUT-49 three steps (d). Circles and diamonds correspond to the compression and decompression of the first cycle respectively and the black line to the compression decompression curves of the second cycle. 
In both cases the first part of the intrusion curve $(<1 \mathrm{MPa})$ corresponds to the compaction of the powder and filling of the interparticular porosity. Subsequently, there is a second step corresponding to a total volume variation of $\sim 1.5 \mathrm{~mL} \cdot \mathrm{g}^{-1}$ and $2.3 \mathrm{~mL} \cdot \mathrm{g}^{-1}$ for DUT-48 and DUT-49, respectively. This is assigned to a contraction of the MOFs since the non-wetting Hg cannot penetrate the mesoporosity of both solids at this pressure. Thus, we assign this change in volume to the contraction of DUT-48 and DUT-49 at $\sim 65 \mathrm{MPa}$ and $\sim 35 \mathrm{MPa}$, respectively. Interestingly, Figure 5 shows a step-wise compression of the two solids. DUT-48 exhibits first increase of the mercury intruded volume associated with a unit cell contraction of $\sim 46.7 \%$ followed by a stepped one leading to a total unit cell contraction of $63.7 \%$. The compression of DUT-49 is even more progressive with the existence of 3 intermediate steps associated with a subsequent unit cell contraction of $13.2 \%, 22.1 \%$ and $48.2 \%$ before reaching a total compression of $71.8 \%$ of the unit cell volume. These experimental findings support the predictions for both solids in terms of transition pressures (higher for DUT-48) and show slightly larger volume changes (similar for both MOFs) but within the margin of error of the method, since a generic force field was used to describe the flexibility of the MOF architectures, and the pressure differences involved here are very small compared to the usual GPa scale of mechanical properties of stiff materials. The $\mathrm{Hg}$ intrusion measurements reveal the structural transitions are irreversible once the applied pressure is released and post mortem PXRD and SEM analysis (Figure S7-9, Figure S17-18) show amorphization and deformation of the crystals suggesting that structural compression beyond the contracted phases occurs.

The so-observed pressure-induced behavior for the two solids corresponds to the scenario of a shock-absorber due to the irreversibility of the structural conversion. ${ }^{3,43}$ From the transition pressures and associated volume changes experimentally evidenced, we can derive work energies 
$(\mathrm{W}=\mathrm{P} \times \Delta \mathrm{V})$ of $85{\mathrm{~J} . \mathrm{g}^{-1}}^{-1}$ and $106{\mathrm{~J} . \mathrm{g}^{-1}}$ for DUT-48 and DUT-49, respectively. These performances are even more attractive than that of the best MOF materials reported so far for such an application (see Table 2). ${ }^{3,43,46}$ 
Table 2 . Comparison of the pressure-induced behaviors and the energy works associated with the structural switching of different MOF materials reported in the literature.

\begin{tabular}{|c|c|c|c|}
\hline MOF Material & Behavior & Work $\left({\mathrm{J} . g^{-1}}^{-1}\right)$ & Reference \\
\hline Silicate & \multirow{5}{*}{$\begin{array}{l}\text { Reversible } \\
\text { nano-spring }\end{array}$} & 11 & 47 \\
\hline ZIF-8-water & & 13.3 & 48 \\
\hline MIL53(Cr)-BDC & & 16 & 8 \\
\hline MIL-47(V)-BDC & & 33 & 17 \\
\hline MIL-53(Al)-FA & & 60 & 16 \\
\hline MIL-53(Al)-BDC & \multirow{4}{*}{$\begin{array}{c}\text { Irreversible } \\
\text { nano shock absorber }\end{array}$} & 7 & 3 \\
\hline MIL-53(Ga)-FA & & 9 & 43 \\
\hline DUT-48 & & 85 & This work \\
\hline DUT-49 & & 106 & This work \\
\hline
\end{tabular}




\section{CONCLUSIONS}

In this study we have synthesized and characterized DUT-48, a highly porous MOF isoreticular

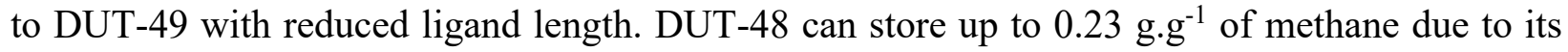
high BET area and pore volume. In contrast to DUT-49, DUT-48 does not undergo a structural transition upon adsorption of nitrogen or $n$-butane.

We have analyzed the adsorption energetics by microcalorimetry and GCMC simulations and determined that the adsorption energy gained by contraction of the pores in DUT-48 is not sufficient for compensating the energy required for this transition thus preventing an adsorptioninduced structural transition. As a result, neither guest-assisted structural contraction or NGA is observed in DUT-48. Furthermore, we have analyzed the mechanical behavior of DUT-48 and DUT-49 by MD and DFT simulations and found that it takes approximately three-times the stress to contract DUT-48 which we relate to the shorter ligand length. In addition, this trend was confirmed by mercury intrusion experiments showing that a higher pressure is required for DUT48 (65 MPa) than DUT-49 (35 MPa) to induce a structural transition. Furthermore, the very high measured work energies of $85{\mathrm{~J} . \mathrm{g}^{-1}}^{1}$ and $106 \mathrm{J.g}^{-1}$ for DUT-48 and DUT-49, respectively, highlight their outstanding potential as future shock-absorbers.

The presented findings provide fundamental insight into structural transition in DUT-49 type structures and importantly link external hydrostatic compression to the energetics of internal adsorption-induced contraction. 


\section{ASSOCIATED CONTENT}

\section{Supporting Information.}

The following files are available free of charge.

Detailed supporting information including additional data and comprehensive experimental and simulation methodology (PDF)

\section{AUTHOR INFORMATION}

\section{Corresponding Author}

*E-mail: stefan.kaskel@chemie.tu-dresden.de

\section{Author Contributions}

The manuscript was written through contributions of all authors. All authors have given approval to the final version of the manuscript. $¥$ These authors contributed equally.

\section{ACKNOWLEDGMENT}

This project has received funding from the European Research Council (ERC) under the European Union's Horizon 2020 research and innovation programme (grant agreement No 742743). J.D.E and F.-X.C acknowledge PSL Research University for funding (project DEFORM, grant ANR-10-IDEX-0001-02), and HPC platforms were provided by a GENCI grant (A0010807069). P. I. and P. L. want to thank the European Union's Horizon 2020 research and innovation programme for funding under the Marie Sklodowska-Curie grant agreement No 641887 (project acronym: DEFNET). The authors thank the ANR/DFG Program FUN for financial support. 


\section{REFERENCES}

1. Occelli, F.; Loubeyre, P.; LeToullec, R., Properties of Diamond under Hydrostatic Pressures up to $140 \mathrm{GPa}$. Nat. Mater. 2003, 2, 151.

2. Wang, J.; Stevens, R., Zirconia-toughened Alumina (ZTA) Ceramics. J. Mater. Sci. 1989, 24 (10), 3421-3440.

3. Yot, P. G.; Boudene, Z.; Macia, J.; Granier, D.; Vanduyfhuys, L.; Verstraelen, T.; Van Speybroeck, V.; Devic, T.; Serre, C.; Ferey, G.; Stock, N.; Maurin, G., Metal-Organic Frameworks as Potential Shock Absorbers: The Case of the Highly Flexible MIL-53(A1). Chem. Commun. 2014, 50 (67), 9462-9464.

4. Maurin, G.; Serre, C.; Cooper, A.; Ferey, G., The New Age of MOFs and of Their Porous-related Solids. Chem. Soc. Rev. 2017, 46 (11), 3104-3107.

5. Burtch, N. C.; Heinen, J.; Bennett, T. D.; Dubbeldam, D.; Allendorf, M. D., Mechanical Properties in Metal-Organic Frameworks: Emerging Opportunities and Challenges for Device Functionality and Technological Applications. Adv. Mater. 2017, 1704124, 10.1002/adma.201704124.

6. Li, W.; Henke, S.; Cheetham, A. K., Research Update: Mechanical Properties of Metal-Organic Frameworks - Influence of Structure and Chemical Bonding. APL Mater. 2014, 2 (12), 123902.

7. Henke, S.; Wharmby, M. T.; Kieslich, G.; Hante, I.; Schneemann, A.; Wu, Y.; Daisenberger, D.; Cheetham, A. K., Pore Closure in Zeolitic Imidazolate Frameworks under Mechanical Pressure. Chem. Sci. 2018, 9, 1654-1660.

8. Beurroies, I.; Boulhout, M.; Llewellyn, P. L.; Kuchta, B.; Férey, G.; Serre, C.; Denoyel, R., Using Pressure to Provoke the Structural Transition of Metal-Organic Frameworks. Angew. Chem. Int. Edit. 2010, 49 (41), 7526-7529.

9. Liu, Y.; Her, J.-H.; Dailly, A.; Ramirez-Cuesta, A. J.; Neumann, D. A.; Brown, C. M., Reversible Structural Transition in MIL-53 with Large Temperature Hysteresis. J. Am. Chem. Soc. 2008, 130 (35), 11813-11818.

10. Li, H.; Hill, M. R., Low-Energy CO2 Release from Metal-Organic Frameworks Triggered by External Stimuli. Accounts Chem. Res. 2017, 50 (4), 778-786.

11. Schneemann, A.; Bon, V.; Schwedler, I.; Senkovska, I.; Kaskel, S.; Fischer, R. A., Flexible Metal-Organic Frameworks. Chem. Soc. Rev. 2014, 43 (16), 6062-6096.

12. Chang, Z.; Yang, D.-H.; Xu, J.; Hu, T.-L.; Bu, X.-H., Flexible Metal-Organic Frameworks: Recent Advances and Potential Applications. Adv. Mater. 2015, 27 (36), 5432-5441.

13. Vanduyfhuys, L.; Rogge, S. M. J.; Wieme, J.; Vandenbrande, S.; Maurin, G.; Waroquier, M.; Van Speybroeck, V., Thermodynamic Insight into Stimuli-responsive Behaviour of Soft Porous Crystals. Nat. Commun. 2018, 9 (1), 204.

14. Su, Z.; Miao, Y.-R.; Zhang, G.; Miller, J. T.; Suslick, K. S., Bond Breakage under Pressure in a Metal Organic Framework. Chem. Sci. 2017, 8 (12), 8004-8011.

15. Öhrström, L., Framework Chemistry Transforming our Perception of the Solid State. ACS Cent. Sci. 2017, 3 (6), 528-530.

16. Yot, P. G.; Vanduyfhuys, L.; Alvarez, E.; Rodriguez, J.; Itie, J.-P.; Fabry, P.; Guillou, N.; Devic, T.; Beurroies, I.; Llewellyn, P. L.; Van Speybroeck, V.; Serre, C.; Maurin, G., Mechanical Energy Storage Performance of an Aluminum Fumarate MetalOrganic Framework. Chem. Sci. 2016, 7 (1), 446-450. 
17. Yot, P. G.; Ma, Q.; Haines, J.; Yang, Q.; Ghoufi, A.; Devic, T.; Serre, C.; Dmitriev, V.; Ferey, G.; Zhong, C.; Maurin, G., Large Breathing of the MOF MIL-47(VIV) under Mechanical Pressure: A Joint Experimental-modelling Exploration. Chem. Sci. 2012, 3 (4), 1100-1104.

18. Rodriguez, J.; Beurroies, I.; Loiseau, T.; Denoyel, R.; Llewellyn, P. L., The Direct Heat Measurement of Mechanical Energy Storage Metal-Organic Frameworks. Angew. Chem. Int. Edit. 2015, 54 (15), 4626-4630.

19. Bousquet, D.; Coudert, F.-X.; Boutin, A., Free Energy Landscapes for the Thermodynamic Understanding of Adsorption-induced Deformations and Structural Transitions in Porous Materials. J. Chem. Phys. 2012, 137 (4), 044118.

20. Neimark, A. V.; Coudert, F.-X.; Triguero, C.; Boutin, A.; Fuchs, A. H.; Beurroies, I.; Denoyel, R., Structural Transitions in MIL-53 (Cr): View from Outside and Inside. Langmuir 2011, 27 (8), 4734-4741.

21. Ferey, G.; Serre, C., Large Breathing Effects in Three-dimensional Porous Hybrid Matter: Facts, Analyses, Rules and Consequences. Chem. Soc. Rev. 2009, 38 (5), 13801399.

22. Ferey, G.; Serre, C.; Devic, T.; Maurin, G.; Jobic, H.; Llewellyn, P. L.; De Weireld, G.; Vimont, A.; Daturi, M.; Chang, J.-S., Why Hybrid Porous Solids Capture Greenhouse Gases? Chem. Soc. Rev. 2011, 40 (2), 550-562.

23. Yot, P. G.; Yang, K.; Guillerm, V.; Ragon, F.; Dmitriev, V.; Parisiades, P.; Elkaïm, E.; Devic, T.; Horcajada, P.; Serre, C.; Stock, N.; Mowat, J. P. S.; Wright, P. A.; Férey, G.; Maurin, G., Impact of the Metal Centre and Functionalization on the Mechanical Behaviour of MIL-53 Metal-Organic Frameworks. Eur. J. Inorg. Chem. 2016, 2016 (27), 4424-4429.

24. Krause, S.; Bon, V.; Senkovska, I.; Stoeck, U.; Wallacher, D.; Többens, D. M.; Zander, S.; Pillai, R. S.; Maurin, G.; Coudert, F.-X.; Kaskel, S., A Pressure-amplifying Framework Material with Negative Gas Adsorption Transitions. Nature 2016, 532 (7599), 348-352.

25. Schaber, J.; Krause, S.; Paasch, S.; Senkovska, I.; Bon, V.; Többens, D. M.; Wallacher, D.; Kaskel, S.; Brunner, E., In Situ Monitoring of Unique Switching Transitions in the Pressure-Amplifying Flexible Framework Material DUT-49 by High-Pressure 129Xe NMR Spectroscopy. J. Phys. Chem. C 2017, 121 (9), 5195-5200.

26. Evans, J. D.; Bocquet, L.; Coudert, F.-X., Origins of Negative Gas Adsorption. Chem 2016, 1 (6), 873-886.

27. Stoeck, U.; Senkovska, I.; Bon, V.; Krause, S.; Kaskel, S., Assembly of MetalOrganic Polyhedra into Highly Porous Frameworks for Ethene Delivery. Chem. Commun. 2015, 51 (6), 1046-1049.

28. Lu, W.; Yuan, D.; Makal, T. A.; Wei, Z.; Li, J.-R.; Zhou, H.-C., Highly Porous Metal-Organic Framework Sustained with 12-connected Nanoscopic Octahedra. Dalton Trans. 2013, 42 (5), 1708-1714.

29. Llewellyn, P. L.; Maurin, G., Gas Adsorption Microcalorimetry and Modelling to Characterise Zeolites and Related Materials. C. R. Chim. 2005, 8 (3), 283-302.

30. Dovesi, R.; Orlando, R.; Erba, A.; Zicovich-Wilson, C. M.; Civalleri, B.; Casassa, S.; Maschio, L.; Ferrabone, M.; De La Pierre, M.; D'Arco, P.; Noël, Y.; Causà, M.; Rérat, M.; Kirtman, B., CRYSTAL14: A Program for the Ab Initio Investigation of Crystalline Solids. Int. J. Quantum Chem. 2014, 114 (19), 1287-1317.

31. Peintinger, M. F.; Oliveira, D. V.; Bredow, T., Consistent Gaussian Basis Sets of Triple-zeta Valence with Polarization Quality for Solid-state Calculations. J. Comput. Chem. 2013, 34 (6), 451-459. 
32. Adamo, C.; Barone, V., Toward Reliable Density Functional Methods Without Adjustable Parameters: The PBE0 Model. J. Chem. Phys. 1999, 110 (13), 6158-6170.

33. Grimme, S., Semiempirical GGA-type Density Functional Constructed with a Longrange Dispersion Correction. J. Comput. Chem. 2006, 27 (15), 1787-1799.

34. Dubbeldam, D.; Calero, S.; Ellis, D. E.; Snurr, R. Q., RASPA: Molecular Simulation Software for Adsorption and Diffusion in Flexible Nanoporous Materials. Mol. Simulat. 2016, 42 (2), 81-101.

35. Rappe, A. K.; Casewit, C. J.; Colwell, K. S.; Goddard, W. A.; Skiff, W. M., UFF, a Full Periodic Table Force Field for Molecular Mechanics and Molecular Dynamics Simulations. J. Am. Chem. Soc. 1992, 114 (25), 10024-10035.

36. Keasler, S. J.; Charan, S. M.; Wick, C. D.; Economou, I. G.; Siepmann, J. I., Transferable Potentials for Phase Equilibria-United Atom Description of Five- and SixMembered Cyclic Alkanes and Ethers. J. Phys. Chem. B 2012, 116 (36), 11234-11246.

37. Bureekaew, S.; Amirjalayer, S.; Tafipolsky, M.; Spickermann, C.; Roy, T. K.; Schmid, R., MOF-FF - A Flexible First-principles Derived Force Field for Metal-Organic Frameworks. Phys. Status Solidi B 2013, 250 (6), 1128-1141.

38. Plimpton, S., Fast Parallel Algorithms for Short-Range Molecular Dynamics. $J$. Comput. Phys. 1995, 117 (1), 1-19.

39. Boyd, P. G.; Moosavi, S. M.; Witman, M.; Smit, B., Force-Field Prediction of Materials Properties in Metal-Organic Frameworks. J. Phys. Chem. Lett. 2017, 8 (2), $357-$ 363.

40. Grunker, R.; Bon, V.; Muller, P.; Stoeck, U.; Krause, S.; Mueller, U.; Senkovska, I.; Kaskel, S., A New Metal-Organic Framework with Ultra-high Surface Area. Chem. Commun. 2014, 50 (26), 3450-3452.

41. Stoeck, U.; Krause, S.; Bon, V.; Senkovska, I.; Kaskel, S., A Highly Porous MetalOrganic Framework, Constructed from a Cuboctahedral Super-molecular Building Block, with Exceptionally High Methane Uptake. Chem. Commun. 2012, 48 (88), 10841-10843.

42. Krause, S.; Bon, V.; Senkovska, I.; Többens, D. M.; Wallacher, D.; Pillai, R. S.; Maurin, G.; Kaskel, S., The Effect of Crystallite Size on Pressure Amplification in Switchable Porous Solids. Nat. Commun. 2018, 9 (1), 1573.

43. Ramaswamy, P.; Wieme, J.; Alvarez, E.; Vanduyfhuys, L.; Itie, J.-P.; Fabry, P.; Van Speybroeck, V.; Serre, C.; Yot, P. G.; Maurin, G., Mechanical Properties of a Gallium Fumarate Metal-Organic Framework: A Joint Experimental-modelling Exploration. $J$. Mater. Chem. A 2017, 5 (22), 11047-11054.

44. Eisley, J. G.; Waas, A. M., Forces and Moments. In Analysis of Structures, John Wiley \& Sons, Ltd: 2011; pp 1-33.

45. Shigley, J. E., Shigley's Mechanical Engineering Design. Tata McGraw-Hill Education: 2011.

46. Ortiz, G.; Nouali, H.; Marichal, C.; Chaplais, G.; Patarin, J., Energetic Performances of "ZIF-71-Aqueous Solution" Systems: A Perfect Shock-Absorber with Water. J. Phys. Chem. C 2014, 118 (37), 21316-21322.

47. Tzanis, L.; Nouali, H.; Daou, T. J.; Soulard, M.; Patarin, J., Influence of the Aqueous Medium on the Energetic Performances of Silicalite-1. Mater. Lett. 2014, 115, 229-232.

48. Ortiz, G.; Nouali, H.; Marichal, C.; Chaplais, G.; Patarin, J., Energetic Performances of the Metal-Organic Framework ZIF-8 Obtained using High Pressure Water Intrusion-extrusion Experiments. Phys. Chem. Chem. Phys. 2013, 15, 4888-4891. 
